\title{
Adipose Derived Stem Cells: A New Hero in Treating Fatty Liver Disease
}

\author{
Usama Sharif Ahmad* \\ Queen Mary University of London, UK
}

*Corresponding author: Usama Sharif Ahmad, Centre for Oral Immunobiology and Regenerative Medicine, Institute of Dentistry,

Barts and The London, School of Medicine and Dentistry, Queen Mary University of London, London, UK

\begin{tabular}{|c|c|}
\hline ARTICLE INFO & ABSTRACT \\
\hline Received: 豐 November 17, 2019 & Abbreviations: ADSC: Adipose Derived Stem Cells; NAFLD: Non-Alcoholic Fatty Liver \\
\hline Published: 蔧January 06, 2020 & $\begin{array}{l}\text { Disease; VLDL: Very-Low Density Lipoproteins; HFD: High Fat Diet; ALF: Acute Liver } \\
\text { Failure; PBS: Phosphate Buffered Saline }\end{array}$ \\
\hline
\end{tabular}

Citation: Usama Sharif Ahmad. Adipose Derived Stem Cells: A New Hero in Treating Fatty Liver Disease. Biomed J Sci \& Tech Res 24(2)-2020. BJSTR. MS.ID.004024.

\begin{abstract}
Mini Review
Adipose derived stem cells (ADSCs) are a type of mesenchymal stem cell with the ability to self-renew and differentiate into multiple lineages. In a recent article, Liao et al. [1] showed that ADSC transplantation in rats promoted the reversion of non-alcoholic fatty liver disease by improving liver function. Non-alcoholic fatty liver disease (NAFLD) is the leading cause of mortality increasing year-on-year. It is estimated $30 \%$ of the population in the Western world suffer from NAFLD. Obese individuals are often associated with NAFLD; however, NAFLD has also been linked with a number of metabolic syndromes such as hypertension, diabetes, hyperlipidaemia and hypertriglyceridemia [2]. The histological spectrum of NALFD ranges from steatosis, non-alcoholic steatohepatitis (NASH), fibrosis, cirrhosis and can eventually result in hepatocellular carcinoma (Figure 1). Steatosis begins when the rate of fatty acid input is greater than the rate of fatty acid output. The accumulation of large amount of fat in the liver can result in inflammation which results in NASH. Persistent inflammation leads to scar tissue formation in the liver marking the onset of fibrosis. Cirrhosis is when the liver shrinks in size and the scar tissue begins to replace the normally healthy tissue of the liver [3].
\end{abstract}

The pathophysiology of NAFLD has often been described as a 'two-hit' hypothesis. In the 'first-hit', fatty acid accumulation results in steatosis leading to the 'second-hit', in which inflammation and oxidative stress occur. However, this hypothesis is now obsolete. A better way of understanding the path mechanisms is the 'multiple hit' hypothesis (Figure 1). The increase of free fatty acids uptake causes an increase in fatty acid oxidation, de novo lipogenesis and very-low density lipoproteins (VLDL). This in turn induces NAFLD. Insulin resistance which can be caused by an increase in fatty acid metabolism, adipose tissue inflammation and endoplasmic reticulum stress can also induce NAFLD [4]. The treatment of NAFLD has an immense clinical and economic burden on governments and health organisations across the word. The United Kingdom along with France, Italy and Germany spend a combined value of $€ 35$ billion euros on treating NAFLD annually. The US on the other hand, spends \$103 billion dollars each year [5]. Current treatment of NAFLD include bariatric surgery, weight loss, exercise, insulin-sensitizing agents, antioxidants and miscellaneous agents. In more serious cases such as cirrhosis, a liver transplantation is required -which is set to be the leading cause of transplantation by 2020. Thus, newer and cost-effective ways of treating NAFLD are urgently required [2]. 


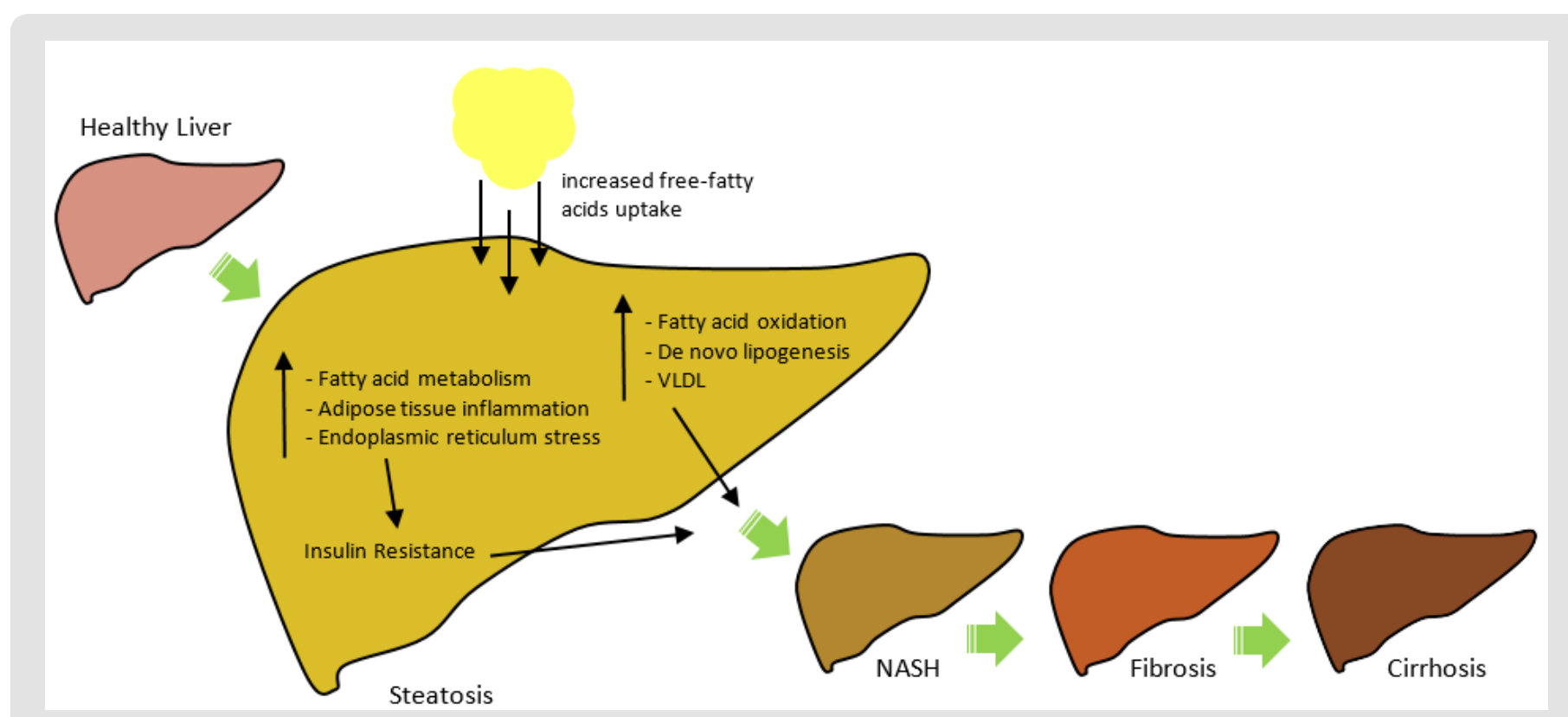

Figure 1: The Spectrum and Pathophysiology of NAFLD.

Note: NAFLD consists of five stages: 1) Steatosis 2) NASH 3) Fibrosis 4) Cirrhosis and 5) Hepatocellular Carcinoma. In the 'multiple hit' hypothesis of NAFLD, an increase in free fatty acids uptake causes an increase in fatty acid oxidation, de novo lipogenesis and VLDL. Insulin resistance can also induce NAFLD.

Adipose derived stem cells (ADSCs) are a type of mesenchymal stem cell. There are many advantages of using ADSCs in regenerative medicine. ADSCs can self-renew, differentiate into multiple lineages and can be easily obtained from abundant adipose tissue. ADSCs can be obtained with very minimal invasive procedure resulting in a high number of cells. It also been reported that ADSCs have immunomodulatory effects and immunosuppressive properties [6]. Liao et al. [1] have shown ADSC transplantation can be used to improve liver function, promote lipid metabolism and exert hepatoprotective effects thereby reversing NAFLD. In this study, thirty-six rats which were fed a high fat diet (HFD) which subsequently developed hepatic steatosis and were equally subdivided into two groups. The control group was treated with phosphate buffered saline (PBS), while the other group received ADSC therapy. Both groups were then fed normal chow. The group observed several positive changes.

ADSC-treated rats had accelerated liver recovery. The liver morphology of these rats had clear signs of recovery. Histological analysis showed the rats had decreased yellow and rough surfaces after 8 weeks, which are typical signs of steatosis. The study also measured biochemical indices of the liver by measuring the serum levels of total bilirubin (TBIL) and alanine aminotransferase (ALT). Following ADSC transplantation, the ADSC-treated rats had decreased levels of ALT and TBIL which suggests an improvement in liver function [1]. In a different study, ADSC transplantation was used to treat acute liver failure (ALF) in rats. Following ADSC transplantation, histological analysis revealed an improvement in liver morphology and function. Moreover, this study also showed decrease serum levels of ALT. They suggest ADSC transplantation may assist in the secretion of factors which help to improve liver function [7]. These studies suggest ADSC transplantation could be a potential treatment for NAFLD, however the safety and mechanisms of such a transplantation are yet to be made clear.

Although the study has shown ADSC transplantation has improved liver function, the change of diet from HFD to normal chow played a massive role. In the control group, the rats had shown signs of liver recovery following a change in diet, but ADSC-treated rats had an obvious accelerated recovery [1]. This shows that changes to diet can over time improve liver function, albeit the time taken is longer. Changes to diet and life-style modifications are the safest and most inexpensive way to treat NAFLD. It is estimated that up to $80 \%$ of individuals who are obese, also suffer from NAFLD [8]. An independent study which examined liver histology from over 500 individuals revealed that the prevalence rates of steatosis in obese individuals was $50 \%$ higher than non-obese individuals, and a further $70 \%$ higher in morbidly obese individuals [3]. In another study, Promrat et al. [9] showed that diet alterations, exercise and behaviour modifications trigged a 7\%-10\% weight loss in obese individuals suffering from NASH. Individuals who had a $7 \%$ weight loss had an improved liver histology.

\section{Acknowledgement}

None.

\section{Conflict of Interest}

No conflict of interest. 


\section{References}

1. Liao N, Pan F, Wang Y, Zheng Y, Xu B, et al. (2016) Adipose tissue-derived stem cells promote the reversion of non-alcoholic fatty liver disease: An in vivo study. International Journal of Molecular Medicine 37(5): 13891396

2. Ahmed M (2015) Non-alcoholic fatty liver disease in 2015. World Journal of Hepatology 7(11): 1450-1459.

3. Fabbrini E, Sullivan S, Klein S (2010) Obesity and non-alcoholic fatty liver disease: Biochemical, metabolic, and clinical implications. Hepatology 51(2): 679-689.

4. Buzzetti E, Pinzani M, Tsochatzis E (2016) The multiple-hit pathogenesis of non-alcoholic fatty liver disease (NAFLD). Metabolism 65(8): 10381048.

\section{ISSN: 2574-1241}

DOI: $10.26717 /$ BJSTR.2020.24.004024

Usama Sharif Ahmad. Biomed J Sci \& Tech Res

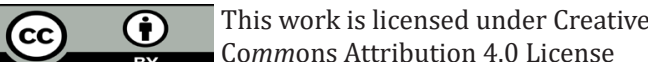

Submission Link: https://biomedres.us/submit-manuscript.php
5. Younossi Z, Blissett D, Blissett R, Henry L, Stepanova M, et al. (2016). The economic and clinical burden of non-alcoholic fatty liver disease in the United States and Europe. Hepatology 64(5): 1577-1586.

6. Tsuji W, Rubin P, Marra K (2014) Adipose-derived stem cells: Implications in tissue regeneration. World Journal of Stem Cells 6(3): 312-321.

7. Chen G, Jin Y, Shi X, Qiu Y, Zhang Y, et al. (2015) Adipose-derived stem cell-based treatment for acute liver failure. Stem Cell Research \& Therapy 6(1): 40.

8. Milić S, Lulić D, Štimac D (2014) Non-alcoholic fatty liver disease and obesity: biochemical, metabolic and clinical presentations. World Journal of Gastroenterology 20(28): 9330-9337.

9. Promrat K, Kleiner D, Niemeier H, Jackvony E, Kearns M, et al. (2010). Randomized controlled trial testing the effects of weight loss on nonalcoholic steatohepatitis. Hepatology 51(1): 121-129.

$\begin{array}{ll}\text { BIOMEDICAL } & \text { Assets of Publishing with us } \\ \text { RESEARCHES } & \text { - Global archiving of articles } \\ \text { - Immediate, unrestricted online access } & \text { - Rigorous Peer Review Process } \\ & \text { - Authors Retain Copyrights }\end{array}$

